\title{
Wearable Computing for Health Care
}

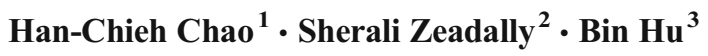

Published online: 4 February 2016

(C) Springer Science+Business Media New York 2016

In recent years, wearable computing has been proliferating at a rapid pace and has enabled a wide range of applications such as mobile communication, sensory integration, behavioral modeling and health care monitoring, etc. Many challenges need to be addressed to enable the integration of multiple sensing/communication technologies for various applications with low energy consumption and low transmission rate. A key design requirement for future wearable health care services is to be able to provide prompt, reliable, secure ubiquitous access to health care services to users and health care providers anytime, anywhere. This special issue explores recent advances in designing, implementing, and deploying novel protocols, architectures, and applications in wearable computing for health care.

This Special issue opens with an article on a big data platform for wearable data in health care, "A Semantic Big Data Platform for Integrating Heterogeneous Wearable Data in Healthcare," authored by Mezghani et al. The diversity, variety, and volume of wearable data related to healthcare make data processing and analytics increasingly difficult. The authors introduce a semantic big data architecture that extends the basic NIST Cloud and Big Data reference architectures with smart mechanisms based on ontology to give meaning to the silos of heterogeneous data. Hence, the authors propose

The Special Issue for this editorial can be found at the following link: http://link.springer.com/journal/10916/39/12/page/1

Han-Chieh Chao

hcchao@gmail.com

\footnotetext{
National Dong Hwa University, Hualien County, Taiwan

University of Kentucky, Lexington, KY, USA

3 Lanzhou University, Lanzhou, China
}

a Wearable Healthcare Ontology that enables the aggregation of the distributed heterogeneous data coming from wearables to make better-informed health related decisions and create new valuable information.

The second article, "Multi-Level MAC-Layer QoS Design in Wireless Body Area Network", by Zhang et al., proposes a multiple level-based QoS design at the MAC layer for a tree topology structured WBAN. The QoS provisioning is divided into three priority levels, i.e., priority of user, priority of data and priority over time. The time slot-based CSMA/CA mechanism in the beacon mode of IEEE 802.15.4 is used for the implementation of the QoS framework. The simulation results demonstrate the effectiveness of the multi-level QoS provisioning in WBAN.

Wearable, non-invasive biosensors, with their portability and low cost, have been applied to a variety of clinical scenarios such as post-traumatic stress disorder, drug addiction, HIV therapy, stress and epilepsy. In the article, "iMStrong: Deployment of a Biosensor System to Detect Cocaine Use", by Carreiro et al., a parameter trajectory description method to analyze the data (such as skin temperature, motion, etc.) collected from bio-sensors is proposed. The authors also presented the detection of drug use by analyzing several parameters. The research is well motivated by the problem of behavior interventions. The authors further prove the feasibility of use the mobile biosensor system in the detection of substance abuse. The authors have collected real data sets. In the future, using mobile bio-sensor system to improve human well-being is a very promising area aimed at reducing health care costs.

The objective of wireless body area sensor networks is to make communication at and near the human body possible in order to measure different body attributes. In the article on wireless body area sensor networks, namely "A Fatigue Measuring Protocol for Wireless Body Area Sensor Networks", Akram et al. propose a fatigue measurement 
protocol for soccer players and soldiers using in-vivo sensors for wireless body area sensor networks. A composite parameter for fatigue measurement is introduced by setting a threshold level for each sensor. Whenever, any sensed data exceeds its threshold level, the players or soldiers are declared to be in a state of fatigue. Moreover, the authors use a vibration pad for the relaxation of fatigued muscles, and then use the vibrational energy by means of a vibration detection circuit to recharge the in-vivo sensors.

In recent years, a wearable heart rate sensing systems have taken various forms, such as wristwatches, wristbands, electronic patches, chest bands, shirts, shoes, and even gloves, chairs, and beds. Among these, systems in clothing form have been regarded as the least invasive and least restraining platform. In the fifth article, "The Effect of Electrode Designs Based on The Anatomical Heart Location for The Noncontact Heart Activity Measurement", by Lee et al., the authors investigate the causes of wide variations in heart rate measurements from the same sensor position among subjects and to enhance the designs of the inductive textile electrode to overcome these variations.

The sixth article, "Data Division Scheme Based on Homomorphic Encryption in WSNs for Health Care", coauthored by Zhang et al., proposes a data division scheme based on homomorphic encryption in a wireless sensor network for health care. Dividing the original data into two or three parts, the scheme provides safe communication and data storage. The scheme also allows for forwarding nodes to upload encrypted sensor data without needing to decrypt the data. As a result, even if a forwarding node is compromised, the attacker will not be able to eavesdrop on the data, resulting in much stronger privacy than existing schemes used in health care. The authors evaluated the performance of their scheme and showed that the robustness of the scheme is good.

The seventh article, "A Lightweight Encryption Scheme Combined with Trust Management for Privacy-preserving in Body Sensor Networks" by Guo et al., designs a secure and privacy-preserving framework combining with multilevel trust management. In their scheme, smart phone resources including computing power and energy can be opportunistically gathered to process the computing-intensive Personal Health Information (PHI) during m-Healthcare emergency with minimal privacy disclosure. In particular, to leverage the PHI privacy disclosure and the high reliability of the PHI process in m-Healthcare emergency, the authors introduce an efficient lightweight encryption for those users whose trust level is low, which is based on mix cipher algorithms as well as plain text and cipher texts, and allow a medical user to decide who can participate in the opportunistic computing to assist in processing the large amount of PHI data. A detailed security analysis and simulation study show that the proposed framework can efficiently achieve user-centric privacy protection in an $\mathrm{m}$-Healthcare system.
The overall demographic profile of current societies points to a significant growth of elderly people. We are also witnessing an increase in life expectancy and an increase in chronic diseases. As a result, there is a need for protection and daily care. Increasing investments in technology, such as Ambient Assisted Living (AAL) solutions, are promoting the quality of life by extending the time people can live in their desired environment. The article on the wearable solutions integration, namely "Integration of Wearable Solutions in AAL Environments with Mobility Support", co-authored by Lorenz et al., proposes the design, deployment, and real testbed of an e-health wearable monitoring system based on the integration of several AAL tools and platforms for monitoring elderly's bio-signals. This solution includes electrocardiography (ECG), respiration rate, beats per minute, body temperature, and fall detention and notification. The paper also describes, in detail, the real pilot study conducted and analyzes some early results concerning the users' quality of experience.

The ninth article, "An Adaptive Sensor Data Segments Selection Method for Wearable Health Care Services", authored by Lai et al. presents the design of an adaptive sensor data feature selection method for wearable health care services, and considered the sensing frequency of the various signals from the human body as well as the data transmission among the devices. The health care service regulates the sensing frequency of devices by considering the overall cloud computing environment and the sensing variations of wearable health care services. The experimental results show that the proposed service can effectively transmit the sensing data and prolong the overall lifetime of health care services.

The tenth article, "Mild Depression Detection of College Students: An EEG-Based Solution with Free Viewing Tasks" by Hu et al., uses a paradigm similar to compare attention bias to emotional images in high risk depressed and normal individuals. For EEG signals, they adopted eight non-linear features besides the classical power and frequency features. Five classifiers including k-nearest-neighbor, Naïve Bayes, Logistic Regression, Support Vector Machine and Random Forest are applied on the combined features for comparison. By analyzing the results of classification, they explore whether the linear and non-linear feature combination could lead to an improvement of performance in depression detection using EEG signal, and which classifier and electrode could best be used for single channel system design with respect to real-time depression detection.

Wearable computing continues to attract a lot of attention thanks to the miniaturization of electronic devices. Wearable healthcare monitoring systems, as an important client of wearable computing technology, have significantly improved. Indeed, the wearable sensors and their surrounding healthcare applications bring a lot of benefits to patients, elderly people and medical staff, thereby improving their daily life quality. In 
the article for wearable health management system, namely "CCS_WHMS: A Congestion Control Scheme for Wearable Health Management System", co-authored by Kafi et al., the authors propose a congestion control scheme CCS WHMS that ensures efficient and fair data delivery when used in the body wearable system part or in the multi-hop inter-bodies wearable ones to get to the final destination. As the congestion detection paradigm is very important in the control process, they perform experimental tests to compare between state of the art congestion detection methods, using MICAz motes, in order to choose the appropriate one for their scheme.

The twelfth article, "Toward a Fault Tolerant Architecture for vital Medical-based Wearable Computing", authored by Mohammadi et al. proposes the design of an architecture for constructing wearable systems in critical medical applications. The proposed architecture is a three-tier one, supporting data flow from body sensors to the cloud. The tiers of this architecture include wearable computers, mobile computing, and mobile cloud computing. One of the features of this architecture is its high fault tolerance due to the nature of its components. Moreover, the required protocols are presented to coordinate the components of this architecture. Finally, the reliability of this architecture is assessed by simulating the architecture and its components.

In the last article, "KinoHaptics: An Automated, Wearable, Haptic Assisted, Physio-therapeutic System for Post-surgery Rehabilitation and Self-care", by Rajanna et al., the authors focus on the development of a health behavior change support system called KinoHaptics, for post-surgery rehabilitation. KinoHaptics is an automated, wearable, haptic assisted, physiotherapeutic system that can be used by a wide variety of users and for various physiological conditions of the patients. The system provides rich and accurate vibrohaptic feedback that can be felt by any user, irrespective of the physiological limitations. KinoHaptics is built to ensure that no injuries are induced during the rehabilitation period. The persuasive nature of the system allows for personal goal-setting, progress tracking, and most importantly lifestyle compatibility.

In closing, we thank all the authors who submitted their research work for consideration for this special issue. We would also like to acknowledge the contribution of many experts in the field who have participated in the review process, and provided helpful suggestions to the authors to improve the quality of their articles.

Finally, we express our gratitude to Dr. Jesse Ehrenfeld, the Editor-in-Chief, for his support and helpful suggestions and comments throughout the preparation of this special issue. We also take this opportunity to thank all staff at Springer for their help and advice.

We hope you will enjoy reading this special issue as much as we did!

\section{Guest editors}

Han-Chieh Chao, National Dong Hwa University, Taiwan

Sherali Zeadally, University of Kentucky, USA

Bin Hu, Lanzhou University, China 